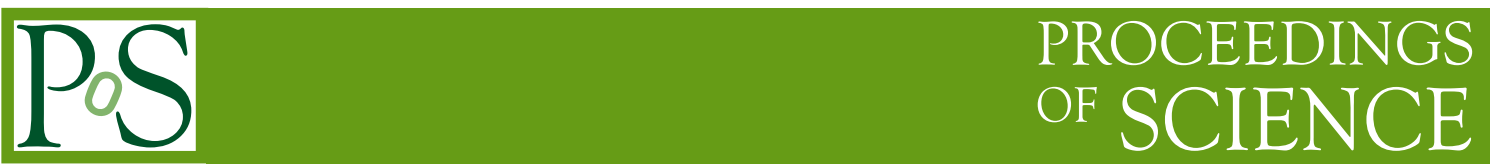

\title{
Inclusive Jet Cross Section at CDF
}

\author{
Régis Lefèvre*† \\ IFAE, Spain \\ E-mail: regisefnal.gov \\ Mario Martínez \\ IFAE, Spain \\ E-mail: mmp@ifae.es
}

This contribution reports on preliminary measurements of the inclusive jet production cross section in $p \bar{p}$ collisions at $\sqrt{s}=1.96 \mathrm{TeV}$ using data collected with CDF corresponding to an integrated luminosity of $385 \mathrm{pb}^{-1}$. Two analyzes are presented: one uses the longitudinally invariant $k_{T}$ algorithm to reconstruct the jets, the other uses the midpoint algorithm. Both are limited to jets with rapidity in the range $0.1<\left|y^{\text {jet }}\right|<0.7$. The measured cross sections are in good agreement with next-to-leading order perturbative QCD predictions after including the non-perturbative corrections necessary to account for underlying event and hadronization effects.

International Europhysics Conference on High Energy Physics

July 21st - 27th 2005

Lisboa, Portugal

\footnotetext{
* Speaker.

$\dagger$ On behalf of the CDF Collaboration. Régis Lefèvre is supported by the EU funding under the RTN contract: HPRN-CT-2002-00292, Probe for New Physics.

$¥$ CDF Collaboration.
} 
The measurement of the inclusive jet production cross section at the Tevatron constitutes an important test of perturbative QCD (pQCD) predictions. As a function of the jet transverse momentum $\left(p_{T}^{j e t}\right)$, the cross section extends over more than eight orders of magnitude. The high $p_{T}^{\text {jet }}$ tail probes distances down to about $10^{-19} \mathrm{~m}$ and is sensitive to new physics [1]. This measurement can also be used to constrain the Parton Distribution Functions (PDFs) at high $x$ and high $Q^{2}$. Run I measurements [2] raised a great interest on an apparent excess at high transverse energy. This excess was finally explained within the Standard Model by increasing the gluon PDF at high $x$ as suggested by global PDF analyzes [3]. Recent PDF sets, such as CTEQ6 [4] and MRST2004 [5], include Run I jet data in their global fits.

The preliminary results presented here use data collected at CDF [6] during Run II and are limited to jets within the range $0.1<\left|y^{j e t}\right|<0.7$. They correspond to an integrated luminosity of $385 \mathrm{pb}^{-1}$, over four times more than for the Run I measurements. In addition, the jet production rate at high $p_{T}^{j e t}$ has significantly increased thanks to the increase of the Tevatron center of mass energy, from $1.8 \mathrm{TeV}$ in Run I to $1.96 \mathrm{TeV}$ in Run II. It has been multiply by a factor five around $600 \mathrm{GeV} / \mathrm{c}$ for instance. Therefore, the $p_{T}^{\text {jet }}$ coverage has been extended by about $150 \mathrm{GeV} / \mathrm{c}$.

New jets algorithms are now explored as the cone algorithm used in Run I is not infrared safe and compromises meaningful comparisons with pQCD calculations [7]. Inclusive jet cross section calculations would be affected at next-to-next-to-leading order. The jets are here reconstructed with the longitudinally invariant $k_{T}$ algorithm [8] or the midpoint algorithm [9].

The latter is still an iterative seed-based cone algorithm but it uses midpoints between pairs of protojets as additional seeds in order to make the clusterization procedure infrared safe. A cone size of $R_{\text {cone }}=0.7$ in the $y-\phi$ plane was used. A merging fraction of $f_{\text {merge }}=0.75$ was used to decide whether overlapping cones have to be merged. To emulate this experimental merging / splitting feature, the corresponding next-to-leading order (NLO) pQCD calculation merges two partons if they are within $R_{\text {cone }} \times R_{\text {sep }}$ of each other and within $R_{\text {cone }}$ of the resulting jet centroid. The parameter $R_{\text {sep }}$ was set to 1.3 according to parton level approximate arguments.

The $k_{T}$ algorithm merges pairs of nearby protojets in order of increasing relative transverse momentum. Inspired by pQCD gluon emissions, it is infrared and collinear safe to all orders in pQCD. Unlike cone based algorithm, it does not include any merging / splitting prescription and allows a well defined comparison with the theory without introducing any arbitrary parameter. On the other hand, it is more sensitive than cone algorithms to soft contributions such as the underlying event or multiple $p \bar{p}$ interactions per bunch crossing. The $k_{T}$ algorithm has a parameter $D$ that approximately controls the size of the jets. To make sure that soft contributions are well understood, the measurement was carried out with three different values: $D=0.5,0.7$, and 1.0.

Regardless of the jet algorithm used, proper comparisons with the theory require corrections for non-perturbative contributions. Those contributions come from the underlying event and the hadronization processes and become more and more important as $p_{T}^{j e t}$ decreases: they could explain the marginal agreement obtained in the DØ Run I study of the inclusive jet cross section using the $k_{T}$ algorithm [10]. The corresponding parton-to-hadron correction was obtained with PYTHIA 6.203 [11] as the ratio of the predicted inclusive jet cross sections at the hadron level on one hand, and at the parton level turning off the interactions between proton and antiproton remnants, on the other hand. A special set of parameters, tuned on Run I CDF data to reproduce the underlying event activity and denoted as PYTHIA-Tune A [12], was used. Tune A has been shown 
to properly describe the jet shapes measured in Run II [13]. The parton-to-hadron level correction was also evaluated with HERWIG 6.4 [14]. The difference between the two Monte Carlos was considered as the systematic uncertainty on the correction.

Figure 1 shows the inclusive jet cross section measured using the $k_{T}$ algorithm with a D parameter of 0.7 and its comparison to theory as well as the parton-to-hadron non-perturbative correction factor. The NLO pQCD cross section was obtained with JETRAD [15] using CTEQ6.1M PDFs [4] and setting the renormalization and factorization scales to $\max \left(p_{T}^{\text {jet }}\right) / 2$. Similarly, figure 2 shows the comparison between data and theory using the midpoint algorithm. In this case, the NLO pQCD cross section was obtained with EKS [16] using $R_{\text {sep }}=1.3$ and CTEQ6.1M PDFs [4], setting the renormalization and factorization scales to $p_{T}^{\text {jet }} / 2$. An additional $\pm 6 \%$ normalization uncertainty associated with the luminosity measurement is not included on both figures. The experimental uncertainties are dominated by the uncertainty on the absolute jet energy scale which is known at the level of $\pm 2 \%$ at low $p_{T}^{\text {jet }}$ and $\pm 3 \%$ at high $p_{T}^{\text {jet }}$ [17]. The main uncertainty in the pQCD prediction comes from the PDFs, especially from the limited knowledge of the gluon PDF at high $x$. The uncertainty on the parton-to-hadron correction factor is also important at low $p_{T}^{\text {jet }}$.

For both the $k_{T}$ and the midpoint algorithms, the measured cross sections are in good agreement with the predictions. In the case of the $k_{T}$ algorithm, similar good agreements between data and theory were obtained using a $D$ parameter of 0.5 and of 1.0 , showing that soft contributions are well under control as their importance depends a lot on the size of the jets. Compared to $D=0.7$, the non-perturbative corrections are for instance about twice smaller for $D=0.5$ and about twice bigger for $D=1.0$.

The speaker would like to acknowledge the EU for its funding under the RTN contract: HPRNCT-2002-00292, Probe for New Physics.
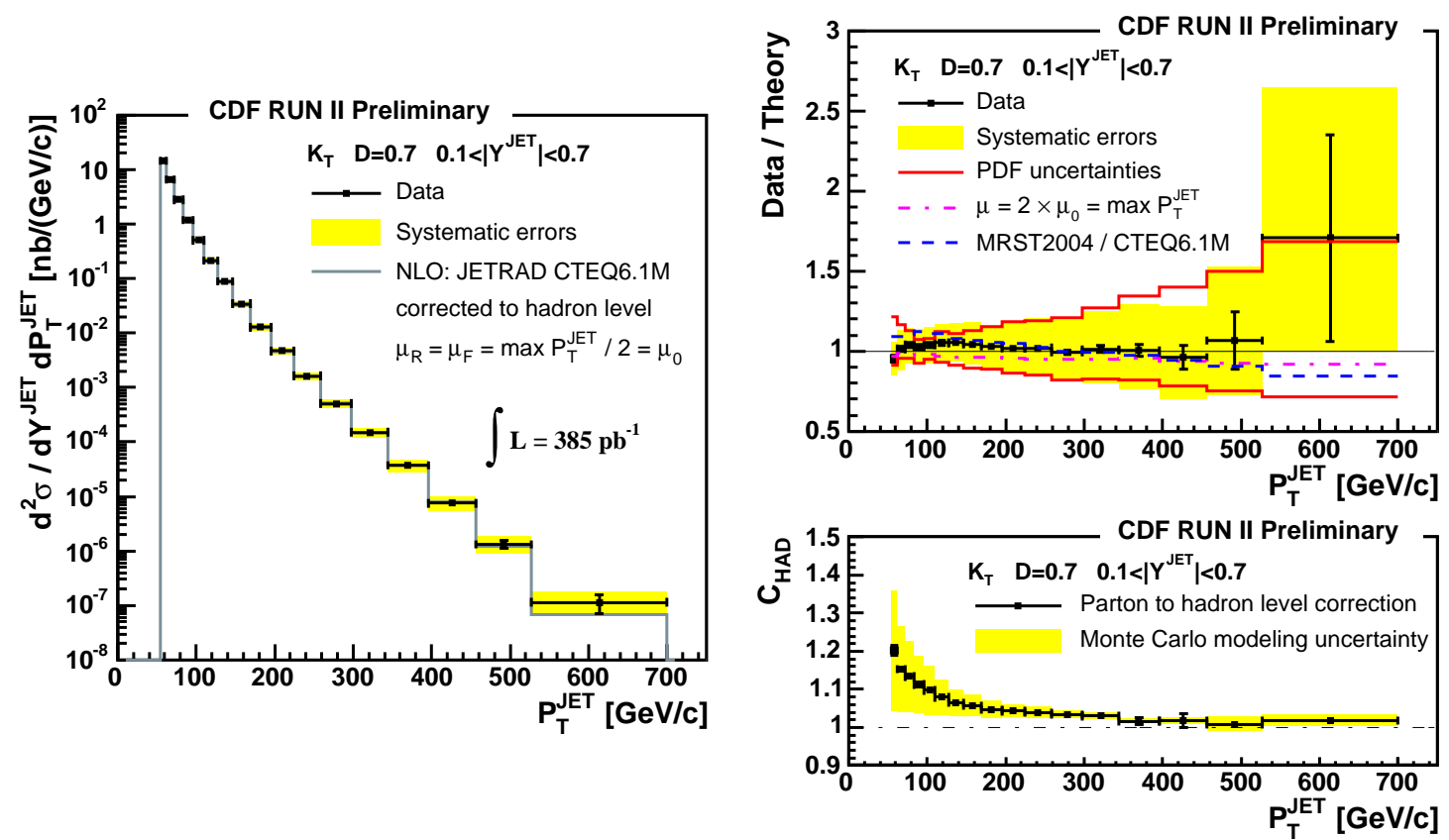

Figure 1: Left: Inclusive jet cross section measured using the $k_{T}$ algorithm with a $D$ parameter of 0.7. Top Right: Ratio of measured and theoretical cross sections. Bottom Right: Parton-to-hadron correction factor. 


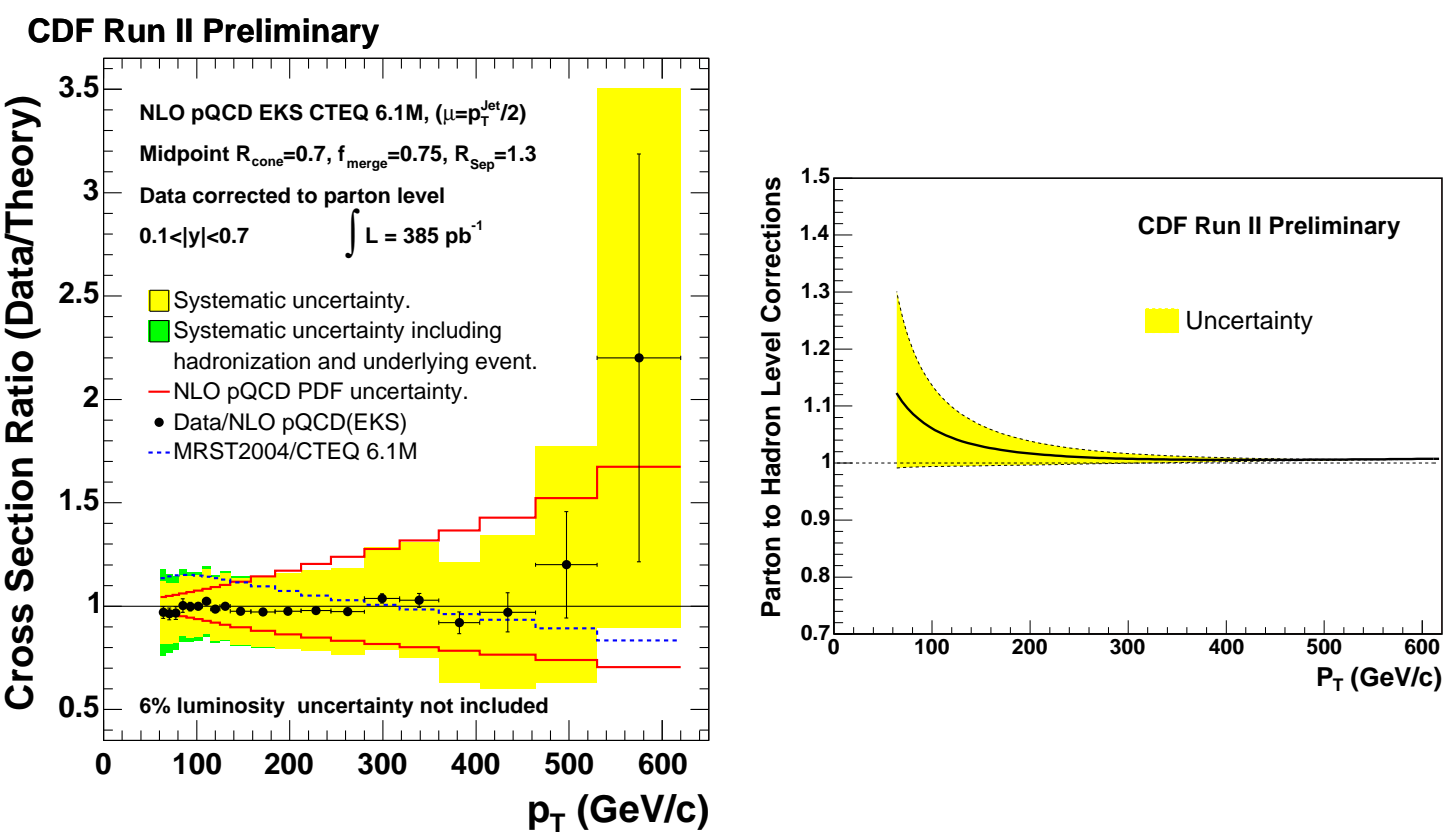

Figure 2: Left: Ratio of measured and theoretical inclusive jet cross sections using the midpoint algorithm. Right: Parton-to-hadron correction factor.

\section{References}

[1] D. Stump et al., JHEP 0310 (2003) 046.

[2] T. Affolder et al. [CDF Collaboration], Phys. Rev. D 64 (2001) 032001 [Erratum-ibid. D 65 (2002) 039903]; B. Abbott et al. [D0 Collaboration], Phys. Rev. Lett. 82 (1999) 2451.

[3] J. Huston et al., Phys. Rev. Lett. 77 (1996) 444.

[4] J. Pumplin et al., JHEP 0207 (2002) 012.

[5] A. D. Martin, R. G. Roberts, W. J. Stirling and R. S. Thorne, Phys. Lett. B 604 (2004) 61.

[6] D. Acosta et al. [CDF Collaboration], Phys. Rev. D 71 (2005) 032001.

[7] M. H. Seymour, Nucl. Phys. B 513 (1998) 269.

[8] S. Catani, Y. L. Dokshitzer, M. H. Seymour and B. R. Webber, Nucl. Phys. B 406 (1993) 187;

S. D. Ellis and D. E. Soper, Phys. Rev. D 48 (1993) 3160.

[9] G. C. Blazey et al., hep-ex/0005012.

[10] V. M. Abazov et al. [D0 Collaboration], Phys. Lett. B 525 (2002) 211.

[11] T. Sjostrand et al., Comput. Phys. Commun. 135 (2001) 238.

[12] T. Affolder et al. [CDF Collaboration], Phys. Rev. D 65 (2002) 092002.

[13] D. Acosta et al. [CDF Collaboration], Phys. Rev. D 71 (2005) 112002, hep-ex/ 0505013.

[14] G. Corcella et al., JHEP 0101 (2001) 010.

[15] W. T. Giele, E. W. N. Glover and D. A. Kosower, Nucl. Phys. B 403 (1993) 633.

[16] S. D. Ellis, Z. Kunszt and D. E. Soper, Phys. Rev. Lett. 64 (1990) 2121.

[17] A. Bhatti et al., hep-ex/0510047. 